\title{
GRAMÁTICA E CULTURA CONTRASTIVA: A ALTERNÂNCIA DAS FORMAS VERBAIS NA INTERAÇÃO TEMPO, MODO, ASPECTO EM ESTUDOS DE TRADUÇÃO
}

\author{
Mônica Maria Guimarães Savedra* \\ Universidade Federal Fluminense
}

\author{
Marina Dupré Lobato** \\ Universidade Federal Fluminense/CNPq
}

\begin{abstract}
Resumo: Em estudos com base na temática de línguas em/de contato, o processo de aquisição de línguas é tratado como um processo relativo, que envolve muitos fatores determinados pelo contexto de aquisição e do uso tópico e dinâmico das línguas em diferentes ambientes comunicativos. Nestes estudos é possível identificar que ao lado de fatores sociolinguísticos e socioculturais, outros fatores, estabelecidos por dimensões conceituais e estruturais, também emanam como relevantes durante o processo de aquisição e uso das línguas. Neste projeto reunimos ao lado do paradigma de tradição sociolinguista o paradigma da Linguística Contrastiva
\end{abstract}

* Formação em Português e Alemão, Universidade do Estado do Rio de Janeiro. Mestrado em Educação, Pontifícia Universidade Católica do Rio de Janeiro. Doutorado em Linguiística, Universidade Federal do Rio de Janeiro. Pós-doutorado em Política Linguística, Universität Duisburg-Essen. Professora Adjunta da Universidade Federal Fluminense, Departamento de Línguas Estrangeiras Modernas - Setor de Alemão. Niterói, Rio de Janeiro, Brasil. E-mail: msavedra@uol.com.br

${ }^{* *}$ Formação em Letras (Língua e Literatura Alemã), Universidade Federal Fluminense. Aluna de mestrado em Estudos de Linguagem, Universidade Federal Fluminense. Niterói, Rio de Janeiro, Brasil. E-mail: marinadupre@gmail.com 
(Kontrastive Linguistik), mais precisamente o da Gramática Contrastiva, em interação com a Cultura Contrastiva (Kulturkontrastive Grammatik). O objeto de nosso estudo é a forma e a funcionalidade das formas verbais em língua alemã e em língua portuguesa. O objetivo deste estudo é identificar e analisar a escolha das formas verbais em traduções de textos literários contemporâneos no par de línguas selecionado com base na alternância dessas formas na interação Tempo, Modo e Aspecto, com especial atenção ao aspecto verbal e a distinção entre aspecto gramatical (Aspekt) e aspecto lexical (Aktionsart).

Palavras-chave: Tradução. Linguística Contrastiva. Usos de formas verbais

\title{
CONTRASTIVE GRAMMAR AND CULTURE: THE ALTERNATION OF VERBAL FORMS IN THE INTERACTION OF TENSE, MOOD AND ASPECT IN TRANSLATION STUDIES
}

\begin{abstract}
In studies based on the theme of languages in/of contact, the process of language acquisition is treated as a relative process, which involves many factors determined by the context of acquisition and of topical and dynamic uses of languages in different communicative environments. In these studies it is possible to identify that next to sociolinguistic and sociocultural factors, other factors established by structural and conceptual dimensions also emanate as relevant to the process of acquisition and use of languages. In this project we united the paradigm of the Sociolinguistics to the paradigm of the Contrastive Linguistics (Kontrastive Linguistik), more precisely of the Contrastive Grammar, in interaction with the Contrastive Culture (Kulturkontrastive Grammatik). The object of our study is the form and the functionality of verbal forms in German and Portuguese. The aim of this study is to identify and to analyze the choice of verbal forms in translations of contemporary literary texts in the pair of languages selected based on the alternation of these forms in the interaction of Tense, Mood and Aspect, with special attention to the verbal aspect and the distinction between grammatical aspect (Aspekt) and lexical aspect (Aktionsart).
\end{abstract}

Keywords: Translation. Contrastive Linguistics. Verbal Forms Uses. 


\section{Apresentação}

Em estudos anteriores desenvolvidos com base na temática de línguas em/de contato, já tivemos a oportunidade de comprovar que o processo de aquisição de línguas é um processo relativo, que envolve muitos fatores determinados pelo contexto de aquisição pelo seu uso tópico e dinâmico das línguas em diferentes ambientes comunicativos: família, sociedade, escola, trabalho. (SAVEDRA, 2007; 2009). Neste sentido, introduzimos a distinção entre bilinguismo e bilingualidade para estudar o tempo e o domínio funcional de uso de cada língua e sua intensidade de interação. Demonstramos que o processo de aquisição de línguas envolve a aquisição de atitudes, valores, crenças e descrenças de determinados grupos (HÖHMANN; SAVEDRA, 2008). Estudamos a estreita relação entre língua, cultura e identidade, a partir do conceito de representação linguística ${ }^{1}$, entendida como um fator sociolinguístico fundamental na determinação de políticas de uso oficial de línguas, bem como na determinação de políticas de manutenção de línguas minoritárias como descrito por Höhmann (2010) na situação do ensino do pomerano no Espírito Santo.

Durante a realização dos estudos acima citados, foi possível identificar que ao lado de fatores sociolinguísticos e socioculturais, outros fatores, estabelecidos por dimensões conceituais e estruturais, também emanam como relevantes durante o processo de aquisição e uso das línguas nas situações de contato analisadas.

Neste trabalho damos continuidade aos estudos anteriores sobre as representações das línguas em contextos de bi- e multilinguismo e pretendemos reunir ao lado deste paradigma de tradição sociolinguista, baseado no uso funcional da língua, o paradigma da Linguística Contrastiva (Kontrastive Liguistik), mas precisamente da Gramática Contrastiva, em interação com a Cultura Contrastiva (Kulturkontrastive Grammatik), como proposto no estudo de Götze, Müller-Liu e Traoré (2009).

Delimitamos a pesquisa aos estudos contrastivos em língua alemã (LA) e língua portuguesa (LP), na variedade do português bra- 
sileiro (LPB). Selecionamos como elemento linguístico de análise o uso das formas verbais (FVs) em LA e em LPB nas traduções em ambas as línguas.

No âmbito de estudos contrastivos em LA e LP, dois trabalhos realizados como teses de doutorado já analisaram o uso das FVs. Ambos delimitaram o enfoque aos tempos verbais do passado. O estudo de Savedra (1994) parte da escolha gramático- discursiva do emprego da flexão verbal em LA e LP em narrativas orais e escritas, experienciadas e elicitadas, produzidas por falantes bilíngues alemão-português e por falantes nativos de alemão, sem domínio da LP, e falantes nativos de LPB sem domínio da LA. O estudo de Battaglia (2008) analisa as características morfológicas, semânticas e pragmáticas dos tempos verbais do passado em textos escritos, de diversas naturezas. A autora contrasta os dois sistemas verbais a partir da descrição na sequência temporal, isto é, na sequência em que os tempos verbais ocorrem nos textos.

$\mathrm{O}$ objeto de nosso estudo é a forma e a funcionalidade das FVs em LA e em LPB. Por forma entende-se a categorização morfológica e regência sintática da flexão verbal em LA e em LP. Por funcionalidade entendem-se os aspectos semântico-pragmáticos do emprego e da função das FVs na tradução do par de línguas em questão, tanto como língua de partida, quanto como língua de chegada. A partir do objeto selecionado, o objetivo geral deste trabalho é identificar a escolha das FVs nas traduções em LA e em LPB e sua alternância na interação Tempo-Modo-Aspecto.

Em um primeiro momento, selecionamos analisar a escolha das FVs nas traduções em LA e LPB, para asserções do modo condicional, discutindo o uso das FVs no âmbito da relação Tempo-Modo-Aspecto, em perspectiva contrastiva. No entanto, identificamos durante a análise do corpus que a noção de aspecto mostrou-se relevante para a escolha da FVs selecionadas nas traduções. Decidimos então direcionar o estudo para a análise do aspecto verbal, especialmente do aspecto durativo/imperfectivo, levando em consideração a distinção entre aspecto gramatical e aspecto lexical (Aktionsart), ou Modo de Ação, sugerida em Koch (2001). 


\section{Gramática e cultura contrastiva: o referencial teórico de base}

Os estudos linguísticos contrastivos realizados na Alemanha tem por base a abordagem comparativa das línguas em contato, como as gramáticas comparativas de Cartagena \& Gauger (1989) e de Zemb (1978). A primeira com estudos comparativos do alemão com o espanhol e a segunda, com o francês. Ambas com o objetivo principal de proporcionar fundamentos linguísticos para o ensino do alemão como língua estrangeira e incentivar a identificação de semelhanças e diferenças entre a língua materna e a língua alvo.

A mesma orientação é seguida no Brasil pelos estudos desenvolvidos no âmbito do projeto Gramática Contrastiva Alemão-Português da USP, descritos por Nomura e Bataglia (2008) em obra que compila algumas pesquisas desenvolvidas no âmbito do projeto.

Mais recentemente, os estudos contrativos passam a considerar a interação gramática-cultura (Kulturkontrastive Grammatik), como proposto por Götze, Müller-Liu e Traoré (2009). A noção de uma gramática contrastiva cultural é introduzida pelos autores como um referencial teórico e metodológico para análise de línguas em contraste não somente pela forma linguística, mas também pelas raízes culturais, tradições e normas, determinadas pelo Weltanschaung ("visão de mundo") de uma respectiva comunidade linguística. Esta nova perspectiva será a perspectiva norteadora deste estudo.

Nesta pesquisa também buscamos subsídios teóricos na sociolinguística cognitiva, como apresentada por Geeraerts, Kristiansen e Peirsman (2010) e por Kristiansen e Dirven (2008). Nestes estudos são propostos modelos teóricos e metodológicos para investigação da variação linguística a partir da interação de modelos cognitivos de construções gramaticais com modelos ideológicos expressos pela representação linguística identificada em determinadas comunidades de fala.

No recente livro organizado por Katny und Socka (2010), os autores discutem a questão da modalidade e da temporalidade 
(Modalität e Temporalität), com base em estudos comparativos de diferentes línguas. Na primeira parte do livro apresentam estudos que privilegiam a interação modalidade e temporalidade exemplificada no contraste da LA com o inglês, com o russo e com o norueguês. Tratam da questão da identidade de tempos e modos verbais e das conjunções como elementos identificadores da integração TempoModo-Aspecto. Na segunda parte, focam a discussão nos verbos modais e na modalidade por eles expressa e, na terceira parte, discutem a lexicalização dos marcadores modais, incluindo a análise contrastiva entre a LA e o polonês.

Além destes estudos em LA, buscamos subsídios teóricos nos trabalhos desenvolvidos em LP sobre a temática de Tempo, Modo e Aspecto.

\section{O uso das formas verbais em LA e em LPB}

A metodologia de investigação que utilizamos é de base descritiva, apoiada em dados empíricos (obras literárias) e na bibliografia especializada da área. O corpus da pesquisa foi constituído por textos literários contemporâneos, de caráter narrativo, escritos em LA e em LPB, bem como suas respectivas traduções. Realizamos um estudo preliminar com base nos seguintes textos:

1. Budapeste de Chico Buarque e tradução de Karin von Schweder-Schreiner (2010)

2. Lavoura arcaica de Raduan Nassar e tradução de Berthold Zilly (2004)

3. Atemschaukel de Herta Müller e tradução de Carola Saavedra (2011)

O referencial teórico que norteou nossa análise abrange gramáticas normativas e descritivas em LPB e em LA (cf. BECHARA, 2006; CUNHA, 2007; CASTILHO, 2010; AZEREDO, 2008; KOCH, 2001; HELBIG, 2002; GÖTZE, 1992; DUDEN, 2009), 
estudos sobre a categoria de tempo, modo e aspecto (cf. TRAVAGLIA, 1994; COMRIE, 1976; KĄTNY, 2010; PETROVA, 2008; RONCARATI, 2009) e ainda estudos contrastivos do alemão e do português (cf. SAVEDRA, 1994; SAVEDRA, 2011; BATAGLIA, 2008; FIGUEIREDO, 2009).

Durante a revisão bibliográfica, definimos alguns parâmetros a serem considerados durante análise do corpus a partir das categorias de Tempo, Modo e Aspecto e revisamos a tabela proposta por Savedra (1994) para a classificação das $\mathrm{FVs}^{2}$ :

\section{Formas Verbais sintáticas e analíticas do Indicativo}

\begin{tabular}{|l|l|}
\hline LA & LP \\
\hline (PRAS) Präsens \\
(PRAT) Präteritum & (PRES) Presente \\
& (IMPF) Pret. Imperfeito \\
& $\begin{array}{l}\text { (PERF) Pret. Perfeito } \\
\text { (+QP) Pret. Mais-q-perfeito } \\
\text { (F PRES) Futuro do Presente } \\
\text { (F PRET) Futuro do Pretérito }\end{array}$ \\
\hline & (PERFC) PERF Composto \\
(PERK) Perfekt & (+QPC) +QP Composto \\
(PLUS) Plusquamperfekt & (FPRESC) FPRES Composto \\
(F1) Futur I & (FPRETC) FPRETComposto \\
(F2) Futur II &
\end{tabular}

\section{Formas Verbais sintáticas e analíticas do Subjuntivo}

\begin{tabular}{|l|l|}
\hline LA & LP \\
\hline (PRAS/K1) Präsens Konjunktiv I & (PRES/S) Presente \\
& (IMPF/S) Pretérito Imperfeito \\
& (F/S) Futuro \\
(PERK/K1) Perfekt Konjunktiv I & (PERFC/S) Pret. Perfeito \\
(PRAT/K2) Präteritum Konjunktiv II & Composto \\
(F1/K2) Futur II Konjunktiv II & (+QPC/S) Pret. Mais-que-perfeito \\
(F2/K2) Futur II Konjunktiv II & composto \\
(PLUS/K2) Plusquamperfekt Konjunktiv II & \\
\hline
\end{tabular}




\section{Perífrases Verbais}

\begin{tabular}{|l|l|}
\hline LA & LP \\
\hline (VPA) Verbalperiphrase-Aspekt & (PVA) perífrases verbais-aspecto \\
(VPM) Verbalperiphrase-Modus & (PVM) perífrases verbais-modo \\
(VPP) Verbalperiphrase-Partzip & (PVP) perífrases verbais-particípio \\
\hline
\end{tabular}

\section{Formas Infinitas}

\begin{tabular}{|l|l|}
\hline LA & LP \\
\hline ( I ) Infinitiv & ( I ) Infinitivo \\
(P1) Partizip I & (G) Gerúndio \\
(P2) Partizip II & (P) Particípio \\
\hline & (IC) Infinitivo composto \\
\hline
\end{tabular}

\section{Aspecto e Modo de Ação}

De acordo com as gramáticas estudadas, o aspecto pode ser definido como uma categoria verbal não dêitica (COMRIE, 1976; TRAVAGLIA, 1994) ou simbólica (CASTILHO, 2010), pois não se refere ao momento da enunciação e não expressa uma localização temporal. Enquanto o tempo verbal representa o tempo externo, o aspecto pode ser considerado como o tempo interno (RONCARATI, 2009; SAVEDRA, 2011), cuja função é expressar o ponto de vista do enunciador sobre o desenvolvimento da ação verbal, ou seja, determinar se a referida ação é concluída ou não concluída, com ou sem enfoque em sua duração. Diferentes aspectos podem ocorrer simultaneamente e, nesse caso, são denominados aspectos compostos (TRAVAGLIA, 1994). Contudo, alguns autores, especialmente em LA, diferenciam o aspecto dependente de formas gramaticais (Aspekt), do aspecto ligado ao significado do lexema verbal, ou modo de ação (Aktionsart), criando ainda outra denominação: aspecto gramatical e aspecto lexical, respectivamente (COMRIE, 1976; KOCH, 2001; HELBIG, 2002; DUDEN, 2009). Sob esse ponto de vista, enquanto o aspecto lexical repre- 
senta o modo como a realidade extralinguística é apreendida, o aspecto gramatical está ligado aos tempos verbais. Nestes casos, uma vez que os valores semânticos relacionados ao verbo e ao contexto não fazem uma oposição gramatical de aspecto, é o significado dos verbos auxiliares que transmite sentidos aspectuais, ou ainda, pelo mesmo motivo, verbos incompatíveis com uma interpretação durativa podem se tornar durativos quando combinados a um verbo auxiliar (CUNHA, 2007; AZEREDO, 2008).

As categorias aspectuais, no entanto, obterão nomenclaturas e subdivisões divergentes. Tendo em vista o objeto deste estudo, investigamos as diferentes definições do aspecto imperfectivo/durativo encontradas durante a revisão bibliográfica.

\section{Aspecto imperfectivo/durativo}

De modo geral, o aspecto imperfectivo apresenta a situação como incompleta, focando na estrutura interna da ação, indicando sua duração e sua conclusão ou não conclusão. Travaglia (1994), ao contrário de outros autores, diferencia o aspecto imperfectivo do durativo, associando-os, respectivamente, à duração e às fases da ação. O imperfectivo apresenta a situação como incompleta e o durativo, como tendo duração contínua ilimitada. Identificamos outros aspectos que se relacionam com os descritos em outros estudos: o iterativo e o habitual (relativo à duração verbal); o não acabado ou começado, o inceptivo, o cursivo e o terminativo (ligados às fases da ação verbal). Em Comrie (1976), a forma imperfectiva pode ser habitual ou contínua, que, por sua vez, pode ainda ser progressiva ou não progressiva. Em Castilho (2010), o aspecto imperfectivo pode ser inceptivo, durativo ou terminativo, dependendo da referência aos pontos de duração, ou ainda iterativo, quando expressa uma repetição durativa. Já em Azeredo (2008), o imperfectivo se refere a um processo concluído ou não concluído denominado progressivo, podendo ser cursivo ou iterativo. Cunha \& Cintra 
(2007) descrevem dois tipos imperfectivos: o durativo, focado na extensão de tempo ocupada pela ação verbal; e o contínuo, focado no processo de desenvolvimento da ação. Em Bechara (2006) o imperfectivo pode ser durativo, iterativo e frequentativo, concluso, inconcluso ou sem traço de conclusão.

Nas gramáticas alemãs, enquanto Götze (1992) e Helbig \& Buscha (2002) adotam os termos "perfectivo" (perfektiv) e "imperfectivo" (imperfektiv), a gramática Duden (2009) opta pela denominação "télico" (telisch) e "atélico" (atelisch), uma vez que perfectivo e imperfectivo servem à categoria aspecto (Aspekt) que, ao contrário da Aktionsart, é expressa por meio de recursos gramaticais regulares. Os verbos atélicos descrevem processos estáticos ou dinâmicos, que não precisam de um ponto final. Esses verbos podem descrever mudanças graduais (verbos atélicos transformativos, em alemão: atelische transformative Verben), ou a não-ocorrência de uma mudança de estado (verbos atélicos não-transformativos, em alemão: atelische intransformative Verben).

Apesar de considerar o aspecto como uma categoria tipicamente verbal, Comrie (1976) e Travaglia (1994) admitem sua presença em formas não-verbais, como substantivos e adjetivos. As variações aspectuais do verbo também podem ser expressas por adjuntos, locuções e expressões adverbiais capazes de gerar diferentes interpretações aspectuais, por afixos, pelo tipo oracional, pela repetição do verbo, pela ênfase entonacional, por preposições, ou ainda pelo complemento e o sujeito do verbo (GÖTZE, 1992; TRAVAGLIA, 1994; HELBIG; 2002; AZEREDO, 2008; DUDEN, 2009; CASTILHO, 2010).

\section{Resultados preliminares}

Quanto à análise do corpus, encontramos dados consistentes no estudo preliminar: 


\begin{tabular}{|c|c|c|c|}
\hline $\begin{array}{l}\text { LPB - } \\
\text { LÍNGUA DE } \\
\text { PARTIDA }\end{array}$ & FV & VF & $\begin{array}{l}\text { LA - } \\
\text { LÍNGUA DE } \\
\text { CHEGADA }\end{array}$ \\
\hline $\begin{array}{l}\text { Teria casado(1) com } \\
\text { ela, na capela de } \\
\text { uma ilha na baía de } \\
\text { Guanabara, se ela não } \\
\text { tivesse me trocado(2) } \\
\text { por um cozinheiro } \\
\text { suíço, e foi(3) então } \\
\text { que fiquei(4) todo } \\
\text { careca, perdi(5) até } \\
\text { os pentelhos, os pêlos } \\
\text { do sovaco, tudo, e o } \\
\text { médico diagnosticou(6) } \\
\text { uma alopecia de fundo } \\
\text { nervoso. }\end{array}$ & $\begin{array}{l}1 \text { (F PRETC) } \\
2 \text { (+ QPC/S) } \\
3 \text { (PERF) } \\
4 \text { (PERF) } \\
5 \text { (PERF) } \\
6 \text { (PERF) }\end{array}$ & $\begin{array}{l}1 \text { (PLUS/K2) } \\
2 \text { (PLUS/K2) } \\
3 \text { - } \\
4 \text { (PRAT) } \\
5 \text { (PRAT) } \\
6 \text { (PRAT) }\end{array}$ & $\begin{array}{l}\text { Ich hätte sie } \\
\text { geheiratet(1), in der } \\
\text { Kapelle auf einer Insel } \\
\text { in der Guanabara-Bucht, } \\
\text { wenn sie mich nicht gegen } \\
\text { einen Schweizer Koch } \\
\text { eingetauscht hätte(2), } \\
\text { und } d a \text { (-) wurde(4) ich } \\
\text { dann vollkommen kahl, } \\
\text { sogar die Schamhaare, die } \\
\text { Haare in der Achselhöhle, } \\
\text { alles verlor(5) } \\
\text { ich, und der Arzt } \\
\text { diagnostizierte(6) eine } \\
\text { nervos bedingte Alopecia. }\end{array}$ \\
\hline
\end{tabular}

Para a FV "foi" (3) em LPB não foi usada uma forma verbal equivalente em LA. O sentido de "foi então" foi dito em LA apenas por meio do advérbio "da".

\begin{tabular}{|c|c|c|c|}
\hline $\begin{array}{l}\text { LPB - } \\
\text { LÍNGUA DE } \\
\text { PARTIDA }\end{array}$ & FV & VF & $\begin{array}{l}\text { LA - } \\
\text { LÍNGUA DE CHEGADA }\end{array}$ \\
\hline $\begin{array}{l}\text { E nós que uma semana } \\
\text { antes havíamos } \\
\text { chegado(7) ao hotel } \\
\text { batendo(8) portas de } \\
\text { táxi e desancando(9) } \\
\text { maleiros, juntos } \\
\text { partimos(10) } \\
\text { lentamente, }\end{array}$ & $\begin{array}{l}7(+\mathrm{QPC}) \\
8(\mathrm{G}) \\
9(\mathrm{G}) \\
10(\mathrm{PERF}) \\
11(\mathrm{G})\end{array}$ & $\begin{array}{l}7 \text { (PLUS) } \\
8 \text { (P1) } \\
9 \text { (P1) } \\
10 \text { (PRAT) } \\
11 \text { (PRAT) }\end{array}$ & $\begin{array}{l}\text { Und wir, die wir eine } \\
\text { Woche zuvor Taxitüren } \\
\text { schlagend(8) und über } \\
\text { Kofferfabrikanten } \\
\text { schimpfend(9) im Hotel } \\
\text { angekommen waren } \\
\text { (7), schleppten (11) } \\
\text { Gepäckstücke }\end{array}$ \\
\hline $\begin{array}{l}\text { arrastando(11) } \\
\text { bagagens repletas de } \\
\text { calhamaços até o ônibus } \\
\text { fretado, no outro lado } \\
\text { da rua. }\end{array}$ & & & $\begin{array}{l}\text { voller Papierberge zum } \\
\text { gecharterten Bus auf der } \\
\text { anderen Straßenseite und } \\
\text { reisten(10) gemeinsam } \\
\text { gemächlich ab(10). }\end{array}$ \\
\hline
\end{tabular}


No que diz respeito ao uso do gerúndio em LPB e do Partizip $I$ em LA e suas respectivas traduções, ainda estamos em fase de análise. Em alguns casos, ambas podem ser equivalentes, como nos exemplos (7) e (8) do trecho citado acima. Entretanto, na realização (10) de LPB, onde o autor utilizou uma forma verbal de gerúndio ("arrastando"), na tradução em LA foi utilizado o Präteritum ("schleppten").

\begin{tabular}{|c|c|c|c|}
\hline $\begin{array}{l}\text { LPB - } \\
\text { LÍNGUA DE PARTIDA }\end{array}$ & FV & $\mathbf{V F}$ & $\begin{array}{l}\text { LA - } \\
\text { LÍNGUA DE CHEGADA }\end{array}$ \\
\hline $\begin{array}{l}\text { (...) e surpreso, e } \\
\text { assustado, senti(1) que } \\
\text { a qualquer momento } \\
\text { eu poderia(2) também } \\
\text { explodir(2) em choro, } \\
\text { me ocorrendo(3) } \\
\text { que seria(4) bom } \\
\text { aproveitar(5) um resto } \\
\text { de embriaguez que não se } \\
\text { deixara(6) espantar(6) } \\
\text { com sua chegada (...) }\end{array}$ & $\begin{array}{l}1 \text { (PERF) } \\
2 \text { (F PRET) } \\
2 \text { (PVM) } \\
3 \text { (G) } \\
4 \text { (F PRET) } \\
5 \text { (I) } \\
6 \text { (+QP) } \\
6 \text { (PVA) }\end{array}$ & $\begin{array}{l}2 \text { (PRAT/ } \\
\text { K2) } \\
2 \text { (VPM) } \\
3 \text { (PRAT) } \\
4 \text { (PRAT) } \\
4 \text { (VPM) } \\
5 \text { - } \\
6 \text { (PLUS) } \\
6 \text { (VPA) }\end{array}$ & $\begin{array}{l}\text { (...) und überrascht und } \\
\text { erschrocken spürte(1) } \\
\text { ich, jeden Augenblick } \\
\text { könnte(2) ich auch in } \\
\text { Tränen ausbrechen(2), } \\
\text { und mir ging(3) durch den } \\
\text { Kopf, ich sollte(4) eine } \\
\text { Resttrunkenheit nutzen(4), } \\
\text { die sich bei seiner Ankunft } \\
\text { nicht hatte(6) verscheuchen } \\
\text { lassen(6) (...) }\end{array}$ \\
\hline
\end{tabular}

No exemplo (3) de Lavoura arcaica, o uso do gerúndio na FV "me ocorrendo" em LPB é compensado pelo uso de uma expressão semanticamente equivalente em LA, "mir ging durch dem Kopf", no Präteritum. Neste caso, ambos denotam o aspecto perfectivo cursivo da ação. No que diz respeito ao uso do condicional, enquanto no exemplo (2) o uso do futuro do pretérito em LPB foi traduzido por uma FV no Präteritum Konjunktiv II em LA, no exemplo (4), foi traduzido por uma FV no Präteritum. 


\begin{tabular}{|c|c|c|c|}
\hline $\begin{array}{l}\text { LA - } \\
\text { LÍNGUA DE PARTIDA }\end{array}$ & FV & VF & $\begin{array}{l}\text { LPB - } \\
\text { LÍNGUA DE CHEGADA }\end{array}$ \\
\hline $\begin{array}{l}\text { (-) Auf dem Heimweg } \\
\text { bin(2) ich in die } \\
\text { Parkmitte, in den runden } \\
\text { Pavillon gegangen(2), } \\
\text { wo an Feiertagen die } \\
\text { Orchester spielten(3). Ich } \\
\text { blieb(4) eine Weile darin } \\
\text { sitzen(4). }\end{array}$ & $\begin{array}{l}1- \\
2 \text { (PERK) } \\
3 \text { (PRAT) } \\
4 \text { (PRAT) } \\
4 \text { (VPA) }\end{array}$ & $\begin{array}{l}1 \text { (G) } \\
2 \text { (PERF) } \\
3 \text { (IMPF) } \\
4 \text { (PERF) } \\
4 \text { (PVA) }\end{array}$ & $\begin{array}{l}\text { Voltando(1) para casa, } \\
\text { fui(2) até o centro do } \\
\text { parque, até o caramanchão } \\
\text { redondo onde as orquestras } \\
\text { se apresentavam(3) nos } \\
\text { dias festivos. Fiquei(4) } \\
\text { algum tempo ali } \\
\text { sentado(4). }\end{array}$ \\
\hline
\end{tabular}

No exemplo (-) de Atemschaukel, há ocorrência de um verbo em LPB (1) sem correspondência em LA. A expressão adverbial "auf dem Heimweg" em LA, foi traduzida em LPB por "voltando para casa”. Em ambas as línguas, as expressões correspondentes indicam o aspecto imperfectivo progressivo da ação - seja em seu uso prototípico, marcado pela FV no gerúndio em LPB, seja pelo uso não prototípico, marcado por uma expressão adverbial em LA. Já no exemplo (4), a perífrase verbal aspectual ocorre no passado em LA e em LPB: "bleiben sitzen" corresponde a "ficar sentado".

\begin{tabular}{|c|c|c|c|}
\hline LA - PARTIDA & VF & FV & LPB - CHEGADA \\
\hline $\begin{array}{l}\text { Ich streifte(5) nach den } \\
\text { fünf Lagerjahren Tag für } \\
\text { Tag durch den Tumult } \\
\text { der Straßen und übte(6) } \\
\text { im Kopf die besten Sätze } \\
\text { für den Fall meiner } \\
\text { Verhaftung. }\end{array}$ & $\begin{array}{l}\text { 5(PRAT) } \\
6 \text { (PRAT) } \\
7-\end{array}$ & $\begin{array}{l}5(\mathrm{IMPF}) \\
6(\mathrm{G}) \\
7(\mathrm{I}) \\
7(\mathrm{PVP})\end{array}$ & $\begin{array}{l}\text { Depois dos cinco anos } \\
\text { no campo de trabalho eu } \\
\text { vagava(5) dia após dia } \\
\text { pelo tumulto das ruas, } \\
\text { ensaiando(6)mentalmente } \\
\text { as melhores frases para o } \\
\text { caso de ser(7) preso(7). }\end{array}$ \\
\hline
\end{tabular}

No exemplo (6), o aspecto imperfectivo da ação marca a simultaneidade entre os verbos (5) "streifen" e "vagar", no passado, e os verbos "üben" e "ensaiar", que em LPB se evidencia pelo uso do gerúndio e em LA, pelo conector "und". Da mesma forma, enquanto a FV (5) está no Präteritum em LA, em LPB ela é expressa pelo pretérito imperfeito, o que dá margem a outra interpretação aspectual. Contudo, a expressão adverbial “Tag für Tag” dá conta 
da noção imperfectiva da ação tanto para a FV (5) quanto para a (6), especialmente em LA. Já “dia após dia”, nesse caso, serve como intensificador.

Na próxima etapa do projeto, continuaremos os estudos teóricos sobre o aspecto em gramáticas alemãs e bibliografia especializada nesta área, com enfoque especial nas formas durativas e no gerúndio. Propomos ainda investigar a pertinência dos estudos sobre Modalidade e Modalização em ambas as línguas.

\section{Notas}

1. Entendemos representação como sendo tudo aquilo que os locutores dizem ou pensam das línguas que falam (ou da maneira como falam) ou das que os outros falam (ou do modo como as falam). As representações são então constituídas pelo conjunto das imagens, das posições ideológicas, ou seja, das crenças que os locutores têm a respeito das línguas em presença e das suas práticas linguísticas, bem como das práticas linguísticas dos outros.

2. Neste momento da pesquisa, utilizamos a categoria de perífrases verbais tanto para a LA, como para a LPB. Para a categoria de Aktionsart, ainda estamos em fase de análise e, portanto, não previmos uma classificação a priori. 


\section{Referências}

AZEREDO, José Carlos de. Gramática Houaiss da Língua Portuguesa. São Paulo: Publifolha, 2008.

BATAGLIA, Maria Helena; NOMURA, Masa. Estudos lingüísticos contrastivos em alemão e português. São Paulo: Ana Blume, 2008.

BECHARA, Evanildo. Moderna Gramática Portuguesa. 37. ed. Rio de Janeiro: Lucerna, 2006.

BUARQUE, Chico. Budapeste. São Paulo: Cia. das Letras, 2003.

. Budapest. Tradução Karin von Schweder-Schreiner. Frankfurt am Main: Fischer Taschenbuch Verlag, 2010.

CARTAGENA, Nelson; GAUGER, Hans-Martin. Vergleichende Grammatik Spanisch-Deutsch. Mannheim: Dudenverlag, 1989. 2 vols.

CASTILHO, Ataliba T. de. Nova gramática do português brasileiro. São Paulo: Contexto, 2010.

COMRIE, Bernard. Aspect. London: Cambridge University Press, 1976.

CUNHA, C.; CINTRA, L. Nova gramática do português contemporâneo. Rio de Janeiro: Lexicon, 2007.

DUDEN. Duden: Die Grammatik. Mannheim: Dudenverlag, 2009. Band 4.

FIGUEIREDO, Sandro. Distribuição e tradução das formas verbais do português e do alemão em prosa literária. Dissertação de mestrado em Letras, Universidade de São Paulo, São Paulo, 2009.

GEERAERTS, D.; KRISTIANSEN, G.; PEIRSMAN, Y. (Ed.). Advances in cognitive sociolinguistics. Berlin: Walter de Gruyter, 2010.

Cad. Trad., Florianópolis, $n^{0}$ especial, p. 299-315, jul./dez. 2014 
GÖTZE, Lutz. Knaurs Grammatik der deutschen Sprache. München: Kaur, 1992.

GÖTZE, L.; MÜLLER-LIU, P.; TRAORÉ, S. Kulturkontrastive Grammatik: Konzepte und Methoden. Fankfurt am Main: Peter Lang, 2009.

HELBIG, G.; BUSCHA, J. Deutsche Grammatik. München: Langenscheidt, 2002.

HÖHMANN, B. Sprachplanung und Spracherhalt innerhalb einer pommerischen Sprachgemeinschaft: Eine soziolinguistische Studie in Espírito Santo. Tese de doutorado, Technische Universität Berlin, Berlin, 2010.

HÖHMANN, B.; SAVEDRA, M. M. G. Spracherhalt und -revitalisierung des Pommerischen in Espírito Santo. Ata do VII Congresso Brasileiro de Professores de Alemão: Fertigkeiten und Kompetenz. Rio de Janeiro: EdUERJ, 2008. p.22.

KATTNY, A.; SOCKA, A. Modalität/Temporalität in kontrastiver und typologischer Sicht. Frankfurt am Main: Peter Lang, 2010.

KOCH, Ingedore Villaça; VILELA, Mário. Gramática da Língua Portuguesa. Coimbra: Almedina, 2001.

KRISTIANSEN, G.; DIRVEN, R. (Ed.) Cognitive Sociolinguistics: language variation, cultural models, social systems. Berlin: Walter de Gruyter, 2008.

MÜLLER, Herta. Atemschaukel. München: Carl Hanser Verlag, 2009.

. Tudo o que tenho levo comigo. São Paulo: Cia. das Letras, 2011.

NASSAR, Raduan. Lavoura arcaica. São Paulo: Cia. das Letras, 2009.

. Das Brot des Patriarchen. Frankfurt am Main: Suhrkamp Verlag, 2004.

PETROVA, Svetlana. Die Interaktion von Tempus und Modus. Heidelberg: Universitätsverlag Winter Heidelberg, 2008.

Cad. Trad., Florianópolis, $n^{0}$ especial, p. 299-315, jul./dez. 2014 
RONCARATI, Cláudia e PEREIRA, Fabiane A. Monteiro. Valores aspectuais e seus efeitos de sentido na expressão da argumentatividade. In: SAVEDRA, M. M. G.; SALGADO, A. C. P. (Orgs.). Sociolinguística no Brasil: uma contribuição dos estudos sobre línguas em/de contato. Rio de Janeiro: 7 Letras, 2009. pp. 29-64.

SAVEDRA, Mônica Maria Guimarães. A marca tempo: tempo interno e tempo externo. In: Bilinguismo e bilingualidade: o tempo passado no discurso em língua portuguesa e língua alemã. Tese de doutorado em linguística, Universidade Federal do Rio de Janeiro, Rio de Janeiro, 1994.

SAVEDRA, M. M. G.; KOELER, E. A equivalência de uso do pretérito pretérito perfeito composto para a língua alemã: Reflexões para o ensino de português como língua estrangeira. In: MEYER, R. M. B.; ALBUQUERQUE, A. (Orgs.). $O$ pretérito perfeito composto no português para estrangeiros: Fronteiras com outras línguas. Rio de Janeiro: Livre Expressão, 2011. p. 119-139.

TRAVAGLIA, Luiz Carlos. O aspecto verbal no português: a categoria e sua expressão. Uberlândia: Editora Universidade Federal de Uberlândia, 1994.

ZEMB, Jean M. Vergleichende Grammatik Französisch-Deutsch. Mannheim: Dudenverlag, 1978. 2 vols.

Recebido em: 12/02/2014

Aceito em: 23/05/2014 